\title{
Relative Telomere Length in Peripheral Blood Cells and Hypertension Risk among Mine Workers: A Case-Control Study in Chinese Coal Miners
}

\author{
Sheng-nan Yu, ${ }^{1}$ Shi-qi Chen, ${ }^{1}$ Guo-quan Fan, ${ }^{2}$ Wei-zhe Pan, ${ }^{1}$ Jin Jia, ${ }^{1}$ Qian Wang, ${ }^{1}$ Li Ma, \\ Ben Li, ${ }^{1}$ Mei Qiang, ${ }^{3}$ Yu-lan Qiu $\left({ }^{1},{ }^{1}\right.$ and Tong Wang $\mathbb{\circledR}^{4}$ \\ ${ }^{1}$ Department of Toxicology, School of Public Health, Shanxi Medical University, Taiyuan, China \\ ${ }^{2}$ Department of Immunology, School of Basic Medical, Shanxi Medical University, Taiyuan, China \\ ${ }^{3}$ Department of Child and Adolescent Health, School of Public Health, Shanxi Medical University, Taiyuan, China \\ ${ }^{4}$ Department of Statistics, School of Public Health, Shanxi Medical University, Taiyuan, China
}

Correspondence should be addressed to Yu-lan Qiu; ylqiu@sxmu.edu.cn and Tong Wang; tongwang@sxmu.edu.cn

Sheng-nan Yu and Shi-qi Chen contributed equally to this work.

Received 7 August 2020; Revised 18 November 2020; Accepted 25 November 2020; Published 7 December 2020

Academic Editor: Caterina Ledda

Copyright ( 2020 Sheng-nan Yu et al. This is an open access article distributed under the Creative Commons Attribution License, which permits unrestricted use, distribution, and reproduction in any medium, provided the original work is properly cited.

\begin{abstract}
Hypertension is a common chronic disease in middle-aged and elderly people and is an important risk factor for many cardiovascular diseases. Its pathogenesis remains unclear. Epidemiological studies have found that the loss of telomere length in peripheral blood cells can increase the risk of coronary heart disease, myocardial infarction, and other diseases. However, a correlation between loss of telomere length and hypertension has not been established. In this study, we aimed to explore the association between telomere length and the risk of essential hypertension (EH) in Chinese coal miners. A case-control study was performed with $215 \mathrm{EH}$ patients and 222 healthy controls in a large coal mining group located in North China. Face-to-face interviews were conducted by trained staff with the necessary medical knowledge. Relative telomere length (RTL) was measured by a quantitative real-time PCR assay using DNA extracted from peripheral blood. In the control group, the age-adjusted RTL was statistically significantly lower in miners performing hard physical labour compared with nonphysical labour $(P=0.043)$. A significantly shorter age-adjusted RTL was found in the control group of participants who consumed alcohol regularly compared with those who do not consume alcohol $(P=0.024)$. Age-adjusted RTL was negatively correlated with body mass index (BMI) and alcohol consumption. Hypertension was also found to be significantly correlated with factors such as age, BMI, alcohol consumption, smoking, and tea consumption. Our results suggest that RTL is associated with hypertension in coal miners.
\end{abstract}

\section{Introduction}

Hypertension is a chronic disease commonly observed in middle-aged and elderly people and is an important risk factor for many cardiovascular diseases [1]. More than $25 \%$ of the Chinese population suffer from hypertension $[2,3]$, resulting in serious health implications within the population, including occupational populations in mining areas [4]. In 2015, health examinations performed in accordance with the Enhanced Coal Workers' Health Surveillance Program (ECWHSP) found that obesity and hypertension were more common in coal-exposed individuals than in unexposed individuals [5]. Recent epidemiological investigations showed that the incidence of cardiovascular disease, especially hypertension, in coal miners is higher than that in the general population [6]. Another study also showed that approximately $50 \%$ of mining-excavator operators developed temporary hypertension within a decade [7].

The telomere is a highly regulated and dynamic complex found at the end of chromosomes consisting of a tract of tandem repeated short DNA repeats and associated protective proteins [8]. Since telomeres progressively shorten with 
increased turnover and chronological age in dividing somatic cells, telomere length may also change and have functional roles in normal and pathophysiological processes [9]. Telomere length is regulated by genetic and environmental factors, and changes in adult telomere length occur chronically over time. Therefore, telomere length is regarded as a marker for biological ageing and age-related diseases such as tumors [10-12]. EH is also an ageing-related chronic pathological condition. Animal studies and epidemiological surveys found that short telomeres may play an important role in the aetiology of hypertension [13-15]. A recent study found that occupational exposure to coal is positively associated with shorter telomere length [16], but the relationship between telomere length and high blood pressure among coal miners is unclear. Shanxi Province is rich in coal resources and has a large number of coal miners. Their health status cannot be ignored.

The aim of this study was to illustrate the role of RTL in occupational coal workers suffering from EH. Additionally, this study also addresses other influencing factors related to $\mathrm{EH}$ in order to promote the health of the workers.

\section{Materials and Methods}

2.1. Study Population and Sample Collection. A large coal mine in the northern Shanxi Province was selected as the research site. A total of 437 coal miners (the average age was $43.97 \pm 8.90)$ were recruited, of which 215 $(44.00 \pm 8.61)$ have $\mathrm{EH}$ and $222(43.95 \pm 9.18)$ were healthy. Questionnaires [17] were administered in the form of faceto-face interviews. The survey included gender, date of birth, education, marital status, place of work, working hours, work intensity, smoking status (current smokers and nonsmokers; current smoking was defined as smoking more than 1 cigarette per day, continuous or cumulative for more than 6 months), tea consumption, and alcohol consumption (consumption was defined as drinking more than once a week and drinking continuously for more than six months). The inclusion criteria of the study people were as follows. (1) The people were aged 18-65 years and had worked in the large coal mine for $\geq 1$ year; case group: diagnosed as essential hypertension; control group: no hypertension. (2) The people participated voluntarily and signed an informed consent. Exclusion criteria were as follows: (1) patients with Parkinson's disease, tumor, and kidney disease (diseases affecting telomere length) at the time of investigation; (2) people with secondary hypertension; and (3) pregnant women or those with incomplete information. Blood pressure was measured after 10 minutes of rest. EH was diagnosed according to the "China Guidelines for the Prevention and Treatment of Hypertension 2010" as systolic blood pressure (SBP) $\geq 140$ $\mathrm{mmHg}$ and diastolic blood pressure (DBP) $\geq 90 \mathrm{mmHg}$. In accordance with the Chinese guidelines on the prevention and control of overweight and obesity in adults, body mass index $(\mathrm{BMI})$ was used to classify thin $(\mathrm{BMI}<18.5)$, normal $(18.5 \leq \mathrm{BMI}<24)$, overweight $(24 \leq \mathrm{BMI}<28)$, and obese $(\mathrm{BMI} \geq 28.00)$ participants. Blood was taken 12 hours after fasting to obtain a DNA separation sample.
2.2. Laboratory Analysis. Peripheral blood samples were collected in ethylenediaminetetraacetic acid tubes after overnight fasting $(>12 \mathrm{~h})$ and kept at $-20^{\circ} \mathrm{C}$ before use. Genomic DNA was isolated from $200 \mu$ l of blood using the QIAamp DNA Blood Mini Kit (QIAGEN, Valencia, CA, USA) following the manufacturer's instructions [18]. In brief, the samples were thawed on ice and mixed briefly by vortexing. Then, the plasma samples were incubated with lysis buffer and proteinase $\mathrm{K}$ at $56^{\circ} \mathrm{C}$ for $10 \mathrm{~min}$. At the final step of isolation, DNA was eluted with $150 \mu$ l of nucleasefree deionized and distilled $\mathrm{H}_{2} \mathrm{O}$. The quantity and purity of the DNA were assayed using a NanoDrop 2000 spectrophotometer (Thermo Scientific, Wilmington, DE, USA), and all DNA samples had OD260/OD280 values of 1.7-2.1. The DNA samples were stored at $-80^{\circ} \mathrm{C}$ until further use. RTL was quantified by real-time polymerase chain reaction (PCR) as described in previous studies $[19,20]$. The primer sequences Tel-F, $5^{\prime}$-CAGCAAGTGGGAAGGTGTAATCC$3^{\prime}$, and Tel-R, $5^{\prime}$-CCCATTCTATCATCAACGGGTACAA$3^{\prime}$, were used to measure the RTL, while the primers 36B4F, 5'-GCTTCTGACACAACTGTGTTCACTAGC-3', and 36B4-R, 5' -CACCAACTTCATCCACGTTCACC-3', were used to amplify the single-copy nuclear 36B4 gene. The assay was performed using the Maxima SYBR Green qPCR Master Mix (TAKARA, Dalian, China) supplied by Line Gene 9660 Real-Time Detection Systems (Bioer, Hangzhou, China). Quantitative PCR was performed under the following conditions: denaturation at $95^{\circ} \mathrm{C}$ for $10 \mathrm{~min}$, followed by 40 cycles of $10 \mathrm{~s}$ at $95^{\circ} \mathrm{C}, 30 \mathrm{~s}$ at $60^{\circ} \mathrm{C}$, and $30 \mathrm{~s}$ at $72^{\circ} \mathrm{C}$. All assays were carried out in triplicates using $20 \mathrm{ng}$ of DNA per $10 \mu \mathrm{l}$ of reaction mixture. The acceptable standard deviation (s.d.) of the triplicate threshold cycle $(\mathrm{Ct})$ values was set at 0.3 . If the result was out of the acceptable range, then the run was repeated for the same sample. RTL was calculated using the following equation: $-\Delta \mathrm{Ct}\left(\Delta \mathrm{Ct}=\mathrm{Ct}_{\mathrm{Tel}}-\mathrm{Ct}_{36 \mathrm{~B} 4}\right)$.

2.3. Statistical Analysis. Data were double-entered into Epi Info version 3.5.1 (CDC, Atlanta, GA, USA), which reduces error in creating electronic data sets before statistical analysis. The difference in the distribution of characteristics between the cases and the controls was evaluated using the chi-square test for categorical variables (age cohorts, education status, work type, work duration, workplace, BMI, alcohol consumption, smoking status, and tea consumption). One-way ANOVA and multiple regression analysis were used to reveal factors that influence RTL in the controls. Crude odds ratios (ORs) and 95\% confidence intervals (CIs) for each of the known risk factors were obtained through univariate logistic regression analysis to evaluate their association with the risk of hypertension. The association between the risk of EH and RTL was estimated using OR and 95\% CIs in unconditional multivariate logistic regression analysis after adjustment for age, education status, work type, work duration, workplace, BMI, alcohol consumption, smoking status, and tea consumption, where appropriate. In the regression models, RTL was analysed as a categorical variable based on a cutoff point at the median values of the controls or continuous variables. All statistical 
analyses were performed with the Statistical Package for the Social Sciences (SPSS, version 24.0 for Windows). All $P$ values reported were two-sided and considered statistically significant at $P<0.05$.

\section{Results}

3.1. Characteristics of the Participants with and without EH. There were no significant differences in age, gender, education, work type, workplace, and tea consumption between the $\mathrm{EH}$ group and the control group $(P>0.05)$. However, there were significant differences in work duration, BMI, alcohol consumption, current smoking, and RTL $(P<0.05)$, as shown in Table 1.

3.2. The Relationship between RTL and Age. A total of 437 peripheral blood samples from $\mathrm{EH}$ and healthy subjects were collected from participants aged between 18 and 65 years. The RTL of peripheral blood cells was determined by RTPCR. The average RTL was $1.1236 \pm 0.8100$ and was significantly negatively correlated with age $(P<0.05)$, indicating that telomeres shortened with age as shown in Figure 1.

The healthy group included 222 participants (200 males and 22 females) aged from 24 to 61 years old $(43.95 \pm 9.18)$ with an average peripheral blood cell RTL of $1.106 \pm 0.689$. The Pearson correlation analysis showed a significant negative correlation between RTL and age in the control group. RTL was negatively correlated with age in both males and females. The male telomere length shortening rate was -0.01 with an $R^{2}$ value of 0.019 , while the female telomere length shortening rate was -0.017 with an $R^{2}$ value of 0.088 . The rate of telomere shortening in the control group was higher in females than in males as shown in Figure 2. The EH group included 215 participants (194 males and 21 females) aged from 23 to 61 years old $(44.00 \pm 8.61)$ with an average peripheral blood cell RTL of $1.142 \pm 0.920$. The Pearson correlation analysis showed no significant correlation between RTL and age in the EH group.

3.3. Influencing Factors of Age-Adjusted RTL in the Control Group. In order to compare the effects of different factors on RTL, using age as a variable, regression analysis was performed on the dependent variable and obtains the residual after standardization as the age-adjusted RTL. After adjusting for age, one-way ANOVA showed that work type and alcohol consumption had significant effects on age-adjusted RTL $(P<0.05)$, while gender, education, work duration, workplace, BMI, current smoking, and tea consumption had no significant effect as shown in Table 2.

3.4. Multiple Regression Analysis of Age-Adjusted RTL. Multiple linear regression analysis of the combined effects of gender, BMI, dietary habits, and other factors on age-adjusted RTL was performed. The results indicated that body mass index $(P<0.01)$, alcohol consumption $(P<0.05)$, and ageadjusted RTL were negatively correlated, while current smoking $(P<0.05)$ was positively correlated with ageadjusted RTL (Table 3 ). Gender, education, tea consumption, and other vocational factors, including work type, work duration, and workplace, were not significantly associated with age-adjusted RTL.

3.5. Univariate Logistic Regression of EH and Other Variables. As outlined in Table 4, univariate logistic regression analysis of 11 factors including age and gender showed that the risk of $\mathrm{EH}$ gradually increases with increasing BMI. The risk of EH in people whose BMI was between 24 to 28 and more than 28 was 11.484 times and 27.077 times more than those whose BMI was less than $18.5(P<0.05)$, respectively. The risk of $\mathrm{EH}$ was also significantly different in alcohol consumption; the risk of $\mathrm{EH}$ was 2.313 times higher in people who drink wine than those who do not $(P<0.05)$. Current smokers have 1.791 times higher risk of hypertension than nonsmokers. The population RTL varied from 0.0199 to 5.1419 and was divided into 3 levels according to the population distribution. The results show a significant difference between longer and shorter RTL. The risk of EH in people with a RTL of 0.57927 to 1.45126 was 0.478 times less than people whose RTL was less than $0.57927(P<0.05)$. The results indicate that telomere length is significantly associated with the risk of $\mathrm{EH}$ and that telomere length is a protective factor against $\mathrm{EH}$.

3.6. Multifactor Logistic Regression of EH and Other Variables. A multifactor logistic regression analysis was performed to consider the interaction between various confounding factors, as shown in Table 5. The results showed that age, BMI, alcohol consumption, smoking (currently smoking), tea consumption, and age-adjusted RTL significantly affected the risk of EH. As BMI is a risk factor for $\mathrm{EH}$, the risk of $\mathrm{EH}$ increased with increasing BMI. Alcohol consumption and smoking are also risk factors for $\mathrm{EH}$; hence, the risk of $\mathrm{EH}$ in people who drink and smoke was 2.103 times and 1.812 times higher, respectively, than those who do not. The results also indicated that tea consumption is a protective factor against $\mathrm{EH}$. The risk of $\mathrm{EH}$ in people who drink tea was 0.609 lower than in those who do not. An analysis of RTL as a categorical variable indicated that RTL may be a predictive factor against $\mathrm{EH}$ as the risk of $\mathrm{EH}$ increased with decreasing telomere length.

\section{Discussion}

In this case-control study, we investigated the influence of various factors on $\mathrm{EH}$ in coal mine workers, particularly the relationship between changes in the RTL in coal miners and $\mathrm{EH}$. Previous studies have shown that the incidence of hypertension in workers is significantly higher than that in the general population $[21,22]$. Souza et al. demonstrated that occupational coal exposure was associated with shortened RTL [16], and a case-control study in India found that shorter RTL was associated with essential hypertension [23]. The telomere length of health care workers (mainly nurses) showed that shift work can lead to shortened telomere length, increased systemic inflammation, and oxidative stress imbalance [24]. The shift work of coal miners in this study may also be an important risk factor for telomere length, which deserves further research. Our results suggest that RTL is associated with hypertension in coal miners. In 
TABLE 1: Characteristics of the participants with and without hypertension.

\begin{tabular}{|c|c|c|c|c|}
\hline Characteristics & Case $(N=215)$ & Control $(N=222)$ & $\chi^{2} / t$ & $P$ value \\
\hline Age & & & 0.969 & 0.809 \\
\hline$\leq 30$ & $18(8.4)$ & $16(7.2)$ & & \\
\hline $30 \sim 40$ & $56(26.0)$ & $57(25.7)$ & & \\
\hline $40 \sim 50$ & $79(36.7)$ & $76(34.2)$ & & \\
\hline$>50$ & $62(28.8)$ & $73(32.9)$ & & \\
\hline Gender & & & 0.002 & 0.960 \\
\hline Male & $194(90.2)$ & $200(90.1)$ & & \\
\hline Female & $21(9.8)$ & $22(9.9)$ & & \\
\hline Education & & & 3.787 & 0.285 \\
\hline College or higher & $16(7.4)$ & $28(12.6)$ & & \\
\hline Senior middle school & $135(62.8)$ & $131(59.0)$ & & \\
\hline Junior middle school & $54(25.1)$ & $56(25.2)$ & & \\
\hline Primary school and less than primary school & $10(4.7)$ & $7(3.2)$ & & \\
\hline Work type & & & 3.397 & 0.183 \\
\hline Hard physical labour & $52(24.2)$ & $71(32.0)$ & & \\
\hline Light physical labour & $113(52.6)$ & $102(45.9)$ & & \\
\hline Nonphysical labour & $50(23.3)$ & $49(22.1)$ & & \\
\hline Work duration & & & 16.018 & $0.007^{*}$ \\
\hline$\leq 1$ year & $5(2.3)$ & $6(2.7)$ & & \\
\hline $2 \sim$ years & $18(8.4)$ & $21(9.5)$ & & \\
\hline $4 \sim$ years & $36(16.7)$ & $70(31.5)$ & & \\
\hline $11 \sim$ years & $27(12.6)$ & $26(11.7)$ & & \\
\hline $16 \sim$ years & $28(13.0)$ & $27(12.2)$ & & \\
\hline$\geq 21$ years & $101(47.0)$ & $72(32.4)$ & & \\
\hline Workplace & & & 3.844 & 0.279 \\
\hline Underground & $52(24.2)$ & $71(32.0)$ & & \\
\hline Underground auxiliary & $68(31.6)$ & $69(31.1)$ & & \\
\hline Ground & $55(25.6)$ & $46(20.7)$ & & \\
\hline Office workers & $40(18.6)$ & $36(16.2)$ & & \\
\hline BMI & & & 34.569 & $<0.001^{*}$ \\
\hline$<18.5$ & $1(0.5)$ & $11(5.0)$ & & \\
\hline $18.5 \sim 24$ & $55(25.6)$ & $94(42.3)$ & & \\
\hline $24 \sim 28$ & $95(44.2)$ & $91(41.0)$ & & \\
\hline$\geq 28$ & $64(29.8)$ & $26(11.7)$ & & \\
\hline Alcohol consumption & & & 17.628 & $<0.001^{*}$ \\
\hline No & $111(51.6)$ & $158(71.2)$ & & \\
\hline Yes & $104(48.4)$ & $64(28.8)$ & & \\
\hline Current smoking & & & 9.141 & $0.002^{*}$ \\
\hline No & $89(41.4)$ & $124(55.9)$ & & \\
\hline Yes & $126(58.6)$ & $98(44.1)$ & & \\
\hline Tea consumption & & & 0.192 & 0.661 \\
\hline No & $138(64.2)$ & $138(62.2)$ & & \\
\hline Yes & $77(35.8)$ & $84(37.8)$ & & \\
\hline RTL & & & 10.685 & $0.005^{*}$ \\
\hline$<0.57927$ & $66(30.7)$ & $44(19.8)$ & & \\
\hline $0.57927 \sim 1.45126$ & $91(42.3)$ & $127(57.2)$ & & \\
\hline$>1.45126$ & $58(27.0)$ & $51(23.0)$ & & \\
\hline
\end{tabular}

${ }^{*} P<0.05$ and the difference is statistically significant. Abbreviations: RTL: relative telomere length; BMI: body mass index. 


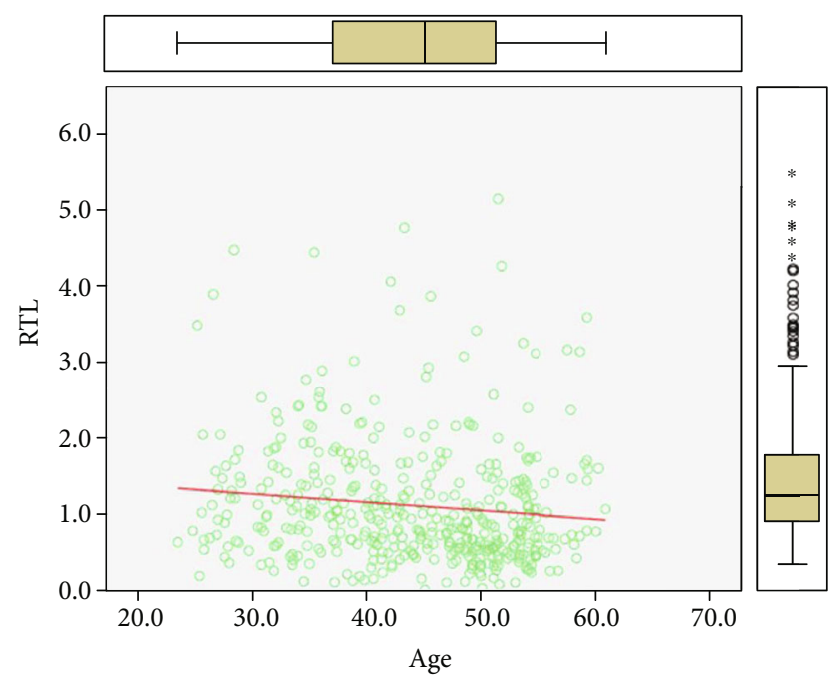

FIGURE 1: Relationship between telomere length and age in the total population. RTL: relative telomere length.

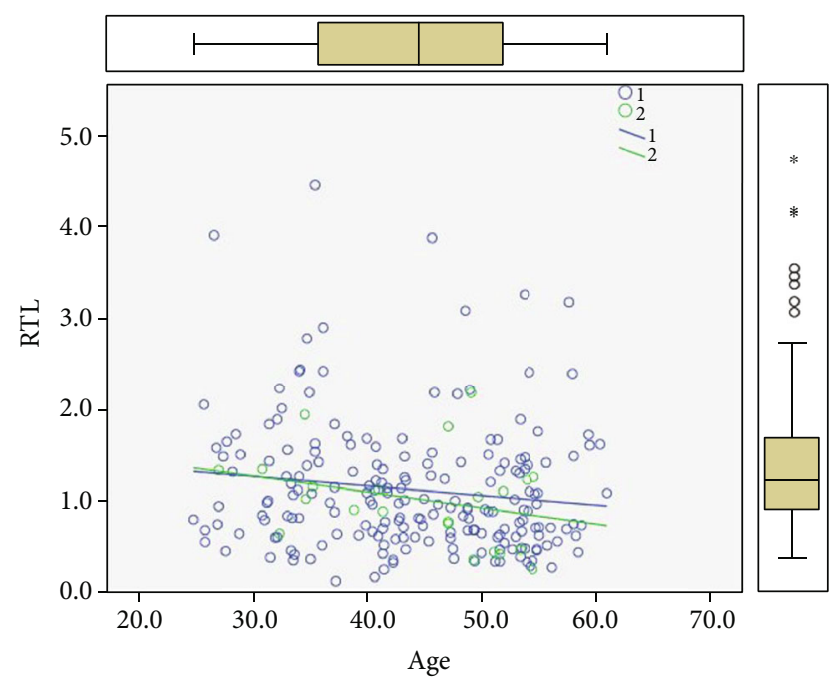

Figure 2: Relationship between telomere length and age in the control group: 1: male; 2 : female.

our study, we divided RTL into three levels according to percentile pitch. Multivariate logistic regression analysis showed that the risk of hypertension in moderate RTL (0.579271.45126 ) is 0.529 times that in shorter RTL. This finding indicates that long RTL is a protective factor in hypertension, which is consistent with previous findings [1]. The reason coal miners have a higher risk of hypertension than the general population may be because the chemical composition of coal is complex and it is accompanied by occupational hazards such as productive dust and toxic heavy metals, toxic and harmful gases, and noise throughout the entire coal production process. Increasing evidence also indicated that toxic and harmful particles enter the body and produce an inflammatory response, which releases reactive oxygen species, leading to an imbalance between oxidation and antioxidant effects in the body, followed by oxidative stress [25]. Exposure to toxic heavy metals, which usually occurs in workers, increases the risk of cardiovascular disease by increasing homocysteine levels, the underlying mechanism may be that high homocysteine leads to changes in the elasticity of the vascular wall, the inhibition of nitric oxide synthesis, and the increase of oxidative stress [26]. A study in mouse models provided evidence that chronic inflammation causes telomere dysfunction and premature ageing [27]. Liu et al.'s study showed that noise is an independent risk factor for hypertension in coal miners [28]. An epidemiological investigation showed that noise can accelerate telomere length loss in peripheral blood leukocytes [29]. Noise, as an environmental stressor, can increase the level of glutathione and harm the body's antioxidant system balance [30]. DNA damage can also be a product of oxidative stress. In our study, the mean age and RTL of the non-EH group were $43.95 \pm 9.18$ and $1.106 \pm 0.689$, respectively. A Chinese study about omethoate showed that the mean RTL of unexposed control group $\leq 40$ years old was $3.03 \pm 0.35$, and the mean RTL of $>40$ years old was $2.97 \pm 0.37$ [31]. The RTL of coal miners is shorter than that of the general population, probably due to the special exposure of the miner occupation. Based on the above information, a hypothesis can be put forward that toxic and harmful particles and noise will affect RTL and eventually lead to higher risk of hypertension in coal miners than the general population. Further research is needed to provide a molecular mechanism that affects telomere length and hypertension in coal miners. In this study, our result shows that the rate of shortening of RTL in healthy control women is significantly higher than that of men, and this conclusion is consistent with many studies $[32,33]$. But there are also opposite conclusions [34], which may be caused by the age span of the study population. We also found that RTL was negatively correlated with age, meaning that RTL gradually shortened with age. Therefore, we should exclude age as a factor when studying RTL within a population. Age-adjusted one-way ANOVA of factors influencing RTL in the control group showed that work type and alcohol consumption had significant effects on age-adjusted RTL. Different work types result in different degrees of possible exposure to coal dust, which explains the influence of work type on age-adjusted RTL. The results of this study also demonstrate that BMI and alcohol consumption were negatively correlated with age-adjusted RTL, suggesting that a healthy BMI and limited alcohol consumption could slow down the rate of RTL shortening in coal miners. The results of multivariate logistic regression analysis showed that age, BMI, alcohol consumption, and current smoking were risk factors for hypertension. The association between BMI and hypertension is consistent with Williams' finding [35]. One study found that the rate of leukocyte telomere shortening is three times greater in active smokers than in nonsmokers. The leukocyte telomere length of previous smokers who had quit smoking before the study was also shorter than in those who never smoked [36]. Our research indicates that current smoking is a risk factor for hypertension, and smoking may cause the shortening of RTL and further induce hypertension. In other words, the shortening of RTL may be an internal influencing mechanism of hypertension. Further studies are required to validate this hypothesis. A systematic review 
TABLE 2: One-way ANOVA of influencing factors of age-adjusted RTL (control group).

\begin{tabular}{|c|c|c|c|c|}
\hline Characteristics & & $N(\%)$ & Age-adjusted RTL & $P$ value \\
\hline \multirow{2}{*}{ Gender } & Male & $200(90.1)$ & $-0.010 \pm 0.869$ & 0.531 \\
\hline & Female & $22(9.9)$ & $-0.130 \pm 0.611$ & \\
\hline \multirow{4}{*}{ Education } & College or higher & $28(12.6)$ & $0.060 \pm 0.903$ & 0.937 \\
\hline & Senior middle school & $131(59.0)$ & $-0.019 \pm 0.892$ & \\
\hline & Junior middle school & $56(25.2)$ & $-0.064 \pm 0.751$ & \\
\hline & Primary school and less than primary schoolwork & $7(3.2)$ & $-0.068 \pm 0.492$ & \\
\hline \multirow{3}{*}{ Work type } & Hard physical labour & $71(32.0)$ & $0.037 \pm 0.822$ & $0.043^{*}$ \\
\hline & Light physical labour & $102(45.9)$ & $-0.165 \pm 0.724$ & \\
\hline & Nonphysical labour & $49(22.1)$ & $0.188 \pm 1.057$ & \\
\hline \multirow{6}{*}{ Work duration } & $\leq 1$ year & $6(2.7)$ & $0.268 \pm 1.671$ & 0.528 \\
\hline & $2 \sim$ years & $21(9.5)$ & $-0.273 \pm 0.738$ & \\
\hline & $4 \sim$ years & $70(31.5)$ & $-0.088 \pm 0.646$ & \\
\hline & $11 \sim$ years & $26(11.7)$ & $0.135 \pm 1.222$ & \\
\hline & $16 \sim$ years & $27(12.2)$ & $-0.021 \pm 0.740$ & \\
\hline & $\geq 21$ years & $72(32.4)$ & $0.034 \pm 0.840$ & \\
\hline \multirow{4}{*}{ Workplace } & Underground & $71(32.0)$ & $-0.180 \pm 0.614$ & 0.080 \\
\hline & Underground auxiliary & $69(31.1)$ & $-0.047 \pm 0.849$ & \\
\hline & Ground & $46(20.7)$ & $0.040 \pm 0.769$ & \\
\hline & Office workers & $36(16.2)$ & $0.258 \pm 1.212$ & \\
\hline \multirow{4}{*}{ BMI } & $<18.5$ & $11(5.0)$ & $0.124 \pm 0.689$ & 0.338 \\
\hline & $18.5 \sim 24$ & $94(42.3)$ & $0.025 \pm 0.884$ & \\
\hline & $24 \sim 28$ & $91(41.0)$ & $-0.010 \pm 0.893$ & \\
\hline & $\geq 28$ & $26(11.7)$ & $-0.297 \pm 0.518$ & \\
\hline \multirow{2}{*}{ Alcohol consumption } & No & $158(71.2)$ & $0.059 \pm 0.904$ & $0.024^{*}$ \\
\hline & Yes & $64(28.8)$ & $-0.222 \pm 0.647$ & \\
\hline \multirow{2}{*}{ Current smoking } & No & $124(55.9)$ & $0.0145 \pm 0.848$ & 0.469 \\
\hline & Yes & $98(44.1)$ & $-0.069 \pm 0.847$ & \\
\hline \multirow{2}{*}{ Tea consumption } & No & $138(62.2)$ & $-0.058 \pm 0.748$ & 0.414 \\
\hline & Yes & $84(37.8)$ & $0.037 \pm 0.989$ & \\
\hline
\end{tabular}

Values are expressed as mean \pm standard deviation. ${ }^{*} P<0.05$ and the difference is statistically significant. Abbreviations: RTL: relative telomere length; BMI: body mass index.

TABLE 3: Multiple regression analysis of age-adjusted RTL.

\begin{tabular}{lccccc}
\hline Characteristics & Upper limit & Lower limit & $t$ & $P$ value & Standardized coefficient $\beta$ \\
\hline Gender & -0.802 & 0.025 & -1.854 & 0.191 & -0.138 \\
Education & -0.251 & 0.129 & -0.633 & 0.065 & -0.050 \\
Work type & -0.322 & 0.098 & -1.051 & 0.527 & -0.097 \\
Work duration & -0.023 & 0.132 & 1.376 & 0.294 & 0.098 \\
Workplace & 0.065 & 0.351 & 2.868 & 0.170 & 0.262 \\
BMI & -0.302 & -0.009 & -2.092 & $0.005^{*}$ & -0.140 \\
Alcohol consumption & -0.578 & -0.056 & -2.392 & $0.038^{*}$ & -0.170 \\
Current smoking & -0.193 & 0.299 & 0.423 & $0.018^{*}$ & 0.031 \\
Tea consumption & -0.134 & 0.347 & 0.870 & 0.673 & 0.061 \\
\hline
\end{tabular}

${ }^{*} P<0.05$ and the difference is statistically significant. Abbreviations: RTL: relative telomere length; BMI: body mass index. 
TABLE 4: Univariate logistic regression of $\mathrm{EH}$ and other variables.

\begin{tabular}{|c|c|c|c|c|c|c|c|}
\hline \multirow{2}{*}{ Characteristics } & & \multirow{2}{*}{$\begin{array}{c}\text { Case } \\
n(\%)\end{array}$} & \multirow{2}{*}{$\begin{array}{c}\text { Control } \\
n(\%)\end{array}$} & \multirow{2}{*}{$P$ value } & \multirow{2}{*}{ OR } & \multicolumn{2}{|c|}{$95 \%$ CI } \\
\hline & & & & & & Upper limit & Lower limit \\
\hline \multirow{4}{*}{ Age } & $\leq 30$ & $18(8.4)$ & $16(7.2)$ & & & & \\
\hline & $30 \sim 40$ & $56(26.0)$ & $57(25.7)$ & 0.729 & 0.873 & 0.405 & 1.882 \\
\hline & $40 \sim 50$ & $79(36.7)$ & $76(34.2)$ & 0.835 & 0.924 & 0.439 & 1.943 \\
\hline & $>50$ & $62(28.8)$ & $73(32.9)$ & 0.465 & 0.755 & 0.355 & 1.604 \\
\hline \multirow{2}{*}{ Gender } & Male & $194(90.2)$ & $200(90.1)$ & & & & \\
\hline & Female & $21(9.8)$ & $22(9.9)$ & 0.960 & 0.984 & 0.524 & 1.847 \\
\hline \multirow{4}{*}{ Education } & College or higher & $16(7.4)$ & $28(12.6)$ & & & & \\
\hline & Senior middle school & $135(62.8)$ & $131(59.0)$ & 0.080 & 1.803 & 0.932 & 3.488 \\
\hline & Junior middle school & $54(25.1)$ & $56(25.2)$ & 0.154 & 1.688 & 0.822 & 3.464 \\
\hline & Primary school and less than primary school & $10(4.7)$ & $7(3.2)$ & 0.117 & 2.500 & 0.796 & 7.853 \\
\hline \multirow{3}{*}{ Work type } & Hard physical labour & $52(24.2)$ & $71(32.0)$ & & & & \\
\hline & Light physical labour & $113(52.6)$ & $102(45.9)$ & 0.069 & 1.513 & 0.968 & 2.365 \\
\hline & Nonphysical labour & $50(23.3)$ & $49(22.1)$ & 0.222 & 1.393 & 0.818 & 2.372 \\
\hline \multirow{6}{*}{ Work duration } & $\leq 1$ year & $5(2.3)$ & $6(2.7)$ & & & & \\
\hline & $2 \sim$ years & $18(8.4)$ & $21(9.5)$ & 0.967 & 1.029 & 0.268 & 3.942 \\
\hline & $4 \sim$ years & $36(16.7)$ & $70(31.5)$ & 0.450 & 0.617 & 0.176 & 2.161 \\
\hline & $11 \sim$ years & $27(12.6)$ & $26(11.7)$ & 0.741 & 1.246 & 0.339 & 4.588 \\
\hline & $16 \sim$ years & $28(13.0)$ & $27(12.2)$ & 0.741 & 1.244 & 0.339 & 4.563 \\
\hline & $\geq 21$ years & $101(47.0)$ & $72(32.4)$ & 0.405 & 1.683 & 0.495 & 5.729 \\
\hline \multirow{4}{*}{ Workplace } & Underground & $52(24.2)$ & $71(32.0)$ & & & & \\
\hline & Underground auxiliary & $68(31.6)$ & $69(31.1)$ & 0.235 & 1.346 & 0.824 & 2.197 \\
\hline & Ground & $55(25.6)$ & $46(20.7)$ & 0.070 & 1.633 & 0.961 & 2.775 \\
\hline & Office workers & $40(18.6)$ & $36(16.2)$ & 0.155 & 1.517 & 0.854 & 2.696 \\
\hline \multirow{4}{*}{ BMI } & $<18.5$ & $1(0.5)$ & $11(5.0)$ & & & & \\
\hline & $18.5 \sim 24$ & $55(25.6)$ & $94(42.3)$ & 0.078 & 6.436 & 0.809 & 51.209 \\
\hline & $24 \sim 28$ & $95(44.2)$ & $91(41.0)$ & $0.021^{*}$ & 11.484 & 1.453 & 90.751 \\
\hline & $\geq 28$ & $64(29.8)$ & $26(11.7)$ & $0.002^{*}$ & 27.077 & 3.325 & 220.507 \\
\hline \multirow{2}{*}{ Alcohol consumption } & No & $111(51.6)$ & $158(71.2)$ & & & & \\
\hline & Yes & $104(48.4)$ & $64(28.8)$ & $<0.001^{*}$ & 2.313 & 1.559 & 3.433 \\
\hline \multirow{2}{*}{ Current smoking } & No & $89(41.4)$ & $124(55.9)$ & & & & \\
\hline & Yes & $126(58.6)$ & $98(44.1)$ & $0.003^{*}$ & 1.791 & 1.226 & 2.617 \\
\hline \multirow{2}{*}{ Tea consumption } & No & $138(64.2)$ & $138(62.2)$ & & & & \\
\hline & Yes & $77(35.8)$ & $84(37.8)$ & 0.661 & 0.917 & 0.621 & 1.352 \\
\hline \multirow{3}{*}{ RTL } & $<0.57927$ & $66(30.7)$ & $44(19.8)$ & & & & \\
\hline & $0.57927 \sim 1.45126$ & $91(42.3)$ & $127(57.2)$ & $0.002^{*}$ & 0.478 & 0.299 & 0.762 \\
\hline & $>1.45126$ & $58(27.0)$ & $51(23.0)$ & 0.311 & 0.758 & 0.444 & 1.296 \\
\hline
\end{tabular}

${ }^{*} P<0.05$ and the difference is statistically significant. Abbreviations: RTL: relative telomere length; BMI: body mass index.

and meta-analysis also found that alcohol consumption was closely related to hypertension, and reducing alcohol intake can effectively reduce blood pressure [37]. Tea consumption and RTL were protective factors which suggest that an active lifestyle intervention could reduce the incidence of hypertension [4]. The effect of tea consumption on blood pressure has been controversial [38], but our findings support the linear mixed effects model analysis which indicates that tea consumption has a positive effect on diastolic blood pressure [39]. We expected that the place of work within this occupational population may have an impact on hypertension and telomere length, but the results do not lead to this conclusion $(P>0.05)$. After further investigation, we found that the occupational groups working in the coal mine area are regularly rotated, which may explain this result.

It should be noted that this study has some limitations. First, this case-control study faces the same inherent limitations seen in retrospective studies such as recall bias and inferior timing of causal association. Second, real-time quantitative PCR was used to determine RTL due to its high specificity. Although high-throughput processing of 
TABLE 5: Multifactor logistic regression of $\mathrm{EH}$ and other variables.

\begin{tabular}{|c|c|c|c|c|c|c|c|}
\hline \multirow{2}{*}{ Characteristics } & & \multirow{2}{*}{$\begin{array}{l}\text { Case } \\
n(\%)\end{array}$} & \multirow{2}{*}{$\begin{array}{c}\text { Control } \\
n(\%)\end{array}$} & \multirow{2}{*}{$P$ value } & \multirow{2}{*}{ OR } & \multicolumn{2}{|c|}{$95 \% \mathrm{CI}$} \\
\hline & & & & & & Upper limit & Lower limit \\
\hline \multirow{4}{*}{ Age } & $\leq 30$ & $18(8.4)$ & $16(7.2)$ & & & & \\
\hline & $30 \sim 40$ & $56(26.0)$ & $57(25.7)$ & 0.246 & 0.557 & 0.208 & 1.496 \\
\hline & $40 \sim 50$ & $79(36.7)$ & $76(34.2)$ & 0.059 & 0.371 & 0.133 & 1.037 \\
\hline & $>50$ & $62(28.8)$ & $73(32.9)$ & $0.010^{*}$ & 0.241 & 0.081 & 0.716 \\
\hline \multirow{2}{*}{ Gender } & Male & $194(90.2)$ & $200(90.1)$ & & & & \\
\hline & Female & $21(9.8)$ & $22(9.9)$ & 0.890 & 0.943 & 0.409 & 2.172 \\
\hline \multirow{4}{*}{ Education } & College or higher & $16(7.4)$ & $28(12.6)$ & & & & \\
\hline & Senior middle school & $135(62.8)$ & $131(59.0)$ & 0.175 & 1.811 & 0.768 & 4.266 \\
\hline & Junior middle school & $54(25.1)$ & $56(25.2)$ & 0.090 & 2.422 & 0.872 & 6.728 \\
\hline & Primary school and less than primary school & $10(4.7)$ & $7(3.2)$ & 0.101 & 3.470 & 0.785 & 15.344 \\
\hline \multirow{3}{*}{ Work type } & Hard physical labour & $52(24.2)$ & $71(32.0)$ & & & & \\
\hline & Light physical labour & $113(52.6)$ & $102(45.9)$ & 0.390 & 1.336 & 0.691 & 2.582 \\
\hline & Nonphysical labour & $50(23.3)$ & $49(22.1)$ & 0.352 & 1.524 & 0.627 & 3.705 \\
\hline \multirow{6}{*}{ Work duration } & $\leq 1$ year & $5(2.3)$ & $6(2.7)$ & & & & \\
\hline & $2 \sim$ years & $18(8.4)$ & $21(9.5)$ & 0.842 & 0.861 & 0.196 & 3.779 \\
\hline & $4 \sim$ years & $36(16.7)$ & $70(31.5)$ & 0.687 & 0.749 & 0.183 & 3.060 \\
\hline & $11 \sim$ years & $27(12.6)$ & $26(11.7)$ & 0.634 & 1.429 & 0.328 & 6.227 \\
\hline & $16 \sim$ years & $28(13.0)$ & $27(12.2)$ & 0.697 & 1.343 & 0.305 & 5.917 \\
\hline & $\geq 21$ years & $101(47.0)$ & $72(32.4)$ & 0.181 & 2.605 & 0.641 & 10.588 \\
\hline \multirow{4}{*}{ Workplace } & Underground & $52(24.2)$ & $71(32.0)$ & & & & \\
\hline & Underground auxiliary & $68(31.6)$ & $69(31.1)$ & 0.429 & 1.310 & 0.672 & 2.554 \\
\hline & Ground & $55(25.6)$ & $46(20.7)$ & 0.332 & 1.466 & 0.677 & 3.175 \\
\hline & Office workers & $40(18.6)$ & $36(16.2)$ & 0.220 & 1.781 & 0.709 & 4.475 \\
\hline \multirow{4}{*}{ BMI } & $<18.5$ & $1(0.5)$ & $11(5.0)$ & & & & \\
\hline & $18.5 \sim 24$ & $55(25.6)$ & $94(42.3)$ & 0.086 & 6.659 & 0.766 & 57.906 \\
\hline & $24 \sim 28$ & $95(44.2)$ & $91(41.0)$ & $0.032^{*}$ & 10.580 & 1.222 & 91.600 \\
\hline & $\geq 28$ & $64(29.8)$ & $26(11.7)$ & $0.004^{*}$ & 23.914 & 2.682 & 213.214 \\
\hline \multirow{2}{*}{ Alcohol consumption } & No & $111(51.6)$ & $158(71.2)$ & & & & \\
\hline & Yes & $104(48.4)$ & $64(28.8)$ & $0.002^{*}$ & 2.103 & 1.311 & 3.375 \\
\hline \multirow{2}{*}{ Current smoking } & No & $89(41.4)$ & $124(55.9)$ & & & & \\
\hline & Yes & $126(58.6)$ & $98(44.1)$ & $0.014^{*}$ & 1.812 & 1.126 & 2.916 \\
\hline \multirow{2}{*}{ Tea consumption } & No & $138(64.2)$ & $138(62.2)$ & & & & \\
\hline & Yes & $77(35.8)$ & $84(37.8)$ & $0.037^{*}$ & 0.609 & 0.383 & 0.970 \\
\hline \multirow{3}{*}{ RTL } & $<0.57927$ & $66(30.7)$ & $44(19.8)$ & & & & \\
\hline & $0.57927 \sim 1.45126$ & $91(42.3)$ & $127(57.2)$ & $0.018^{*}$ & 0.529 & 0.312 & 0.897 \\
\hline & $>1.45126$ & $58(27.0)$ & $51(23.0)$ & 0.551 & 0.827 & 0.443 & 1.544 \\
\hline
\end{tabular}

${ }^{*} P<0.05$ and the difference is statistically significant. Abbreviations: RTL: relative telomere length; BMI: body mass index.

large samples has been widely used, it has become controversial in recent years compared with the traditional DNA blotting because the result of the measurement is a relative value and not an absolute value. Additionally, the resolution and accuracy of this method is lower than those of the conventional method [40] and repeated measurements of the coefficient of variation exceeded $10 \%$.

In conclusion, our results suggest that RTL is associated with $\mathrm{EH}$ in coal miners. We also found that the factors such as BMI, smoking, alcohol consumption, and tea consump- tion were closely associated with $\mathrm{EH}$ in coal miners. This information may provide a scientific basis for preventing hypertension through lifestyle interventions. It also provides a solid foundation to study the pathogenesis of hypertension in mine workers.

\section{Data Availability}

Data used to support the findings of this study are available from the corresponding author upon reasonable request. 


\section{Ethical Approval}

This study was approved by the Ethics Committee of Shanxi Medical University.

\section{Conflicts of Interest}

The author declares that there are no conflicts of interest.

\section{Authors' Contributions}

Sheng-nan Yu and Shi-qi Chen performed data analyses, drafted the manuscript, and finalized and submitted the manuscript. Sheng-nan Yu and Shi-qi Chen performed the DNA extraction and qPCR experiments. Guo-quan Fan performed the DNA extraction. Wei-zhe Pan, Jin Jia, Qian Wang, Ben Li, Li Ma, and Mei Qiang contributed to the data collection and quality control. Yu-lan Qiu and Tong Wang were responsible for the conception and design of the study, collection of data, and drafting and revision of the manuscript. All the authors approved the final version of the manuscript. Sheng-nan Yu and Shi-qi Chen contributed equally to this work.

\section{Acknowledgments}

The authors are thankful for the support of Shanxi Province Graduate Education Innovation Center. Thanks are also due to Shanxi Datong Coal Mine Group General Hospital for providing the epidemiological investigation site. This work was supported by the National Natural Science Foundation of China (Grant number 81872715) and the Research Project Supported by the Shanxi Scholarship Council of China (Grant number 2016-056).

\section{References}

[1] Y. Maida, S. Kyo, N. R. Forsyth et al., "Distinct telomere length regulation in premalignant cervical and endometrial lesions: implications for the roles of telomeres in uterine carcinogenesis," The Journal of Pathology, vol. 210, no. 2, pp. 214-223, 2006.

[2] X. Chen, H. Du, J. Zhang et al., "Adiposity and blood pressure among 55000 relatively lean rural adults in southwest of China," Journal of Human Hypertension, vol. 29, no. 9, pp. 522-529, 2015.

[3] Y. Liu, Y.-Q. Lou, K. Liu et al., "Role of leptin receptor gene polymorphisms in susceptibility to the development of essential hypertension: a case-control association study in a Northern Han Chinese population," Journal of Human Hypertension, vol. 28, no. 9, pp. 551-556, 2014.

[4] P. Zheng, B. Zeng, C. Zhou et al., "Gut microbiome remodeling induces depressive-like behaviors through a pathway mediated by the host's metabolism," Molecular Psychiatry, vol. 21, no. 6, pp. 786-796, 2016.

[5] M. L. Casey, K. B. Fedan, N. Edwards et al., "Evaluation of high blood pressure and obesity among US coal miners participating in the Enhanced Coal Workers' Health Surveillance Program," Journal of the American Society of Hypertension, vol. 11, no. 8, pp. 541-545, 2017.
[6] L. Lei, J. Guo, X. Shi et al., "Mitochondrial DNA copy number in peripheral blood cell and hypertension risk among mining workers: a case-control study in Chinese coal miners," Journal of Human Hypertension, vol. 31, no. 9, pp. 585-590, 2017.

[7] O. Ustinova, V. B. Alekseev, A. N. Rumiantseva, and O. Iav, "Influence of work intensity on development of arterial hypertension in metal-mining workers," Meditsina Truda $i$ Promyshlennaia Ekologiia, vol. 11, pp. 27-31, 2013.

[8] E. H. Blackburn, E. S. Epel, and J. Lin, "Human telomere biology: a contributory and interactive factor in aging, disease risks, and protection," Science, vol. 350, no. 6265, pp. 11931198, 2015.

[9] J. W. Shay, "Telomeres and aging," Current Opinion in Cell Biology, vol. 52, pp. 1-7, 2018.

[10] L. Hjort, R. Vryer, L. G. Grunnet et al., "Telomere length is reduced in 9- to 16-year-old girls exposed to gestational diabetes in utero," Diabetologia, vol. 61, no. 4, pp. 870-880, 2018.

[11] J. L. Sanders, A. L. Fitzpatrick, R. M. Boudreau et al.Leukocyte telomere length is associated with noninvasively measured age-related disease: the Cardiovascular Health Study," The Journals of Gerontology Series A: Biological Sciences and Medical Sciences, vol. 67A, no. 4, pp. 409-416, 2012.

[12] K. A. Biegler, A. K. L. Anderson, L. B. Wenzel, K. Osann, and E. L. Nelson, "Longitudinal change in telomere length and the chronic stress response in a randomized pilot biobehavioral clinical study: implications for cancer prevention," Cancer Prevention Research, vol. 5, no. 10, pp. 1173-1182, 2012.

[13] G. Pérez-Rivero, M. P. Ruiz-Torres, J. V. Rivas-Elena et al., "Mice deficient in telomerase activity develop hypertension because of an excess of endothelin production," Circulation, vol. 114, no. 4, pp. 309-317, 2006.

[14] L. Ma, Y. Li, and J. Wang, "Telomeres and essential hypertension," Clinical Biochemistry, vol. 48, no. 16-17, pp. 1195-1199, 2015.

[15] J. M. Wojcicki, D. Elwan, J. Lin, E. Blackburn, and E. Epel, "Chronic obesity and incident hypertension in Latina women are associated with accelerated telomere length loss over a 1year period," Metabolic Syndrome and Related Disorders, vol. 16, no. 6, pp. 262-266, 2018.

[16] M. R. de Souza, V. F. S. Kahl, P. Rohr et al., "Shorter telomere length and DNA hypermethylation in peripheral blood cells of coal workers," Mutation Research/Genetic Toxicology and Environmental Mutagenesis, vol. 836, pp. 36-41, 2018.

[17] Y. Cui, S.-S. Tian, N. Qiao et al., "Associations of individualrelated and job-related risk factors with nonfatal occupational injury in the coal workers of Shanxi Province: a cross-sectional study," PLoS One, vol. 10, no. 7, article e0134367, 2015.

[18] T.-D. Jeong, Y.-U. Cho, W. Lee, S. Chun, and W. K. Min, “An efficient genomic DNA extraction from whole blood using Nextractor," Clinica Chimica Acta, vol. 435, pp. 14-17, 2014.

[19] N. J. O'Callaghan and M. Fenech, "A quantitative PCR method for measuring absolute telomere length," Biological Procedures Online, vol. 13, no. 1, 2011.

[20] A. J. Montpetit, A. A. Alhareeri, M. Montpetit et al., "Telomere length: a review of methods for measurement," Nursing Research, vol. 63, no. 4, pp. 289-299, 2014.

[21] R. Guo, Z. W. Wang, X. Wang, L. Zhang, Z. Chen, and M. Guo, "Hypertension prevalence for working population in several provinces in China," Chinese Circulation Journal, vol. 35, pp. 734-738, 2014. 
[22] B. Sonkodi, S. Sonkodi, S. Steiner et al., "High prevalence of prehypertension and hypertension in a working population in Hungary," American Journal of Hypertension, vol. 25, no. 2, pp. 204-208, 2012.

[23] C. Bhupatiraju, D. Saini, S. Patkar, P. Deepak, B. Das, and T. Padma, "Association of shorter telomere length with essential hypertension in Indian population," American Journal of Human Biology, vol. 24, no. 4, pp. 573-578, 2012.

[24] C. Ledda, C. Loreto, and V. Rapisarda, "Telomere length as a biomarker of biological aging in shift workers," Applied Sciences, vol. 10, no. 8, p. 2764, 2020.

[25] R. P. Barnes, E. Fouquerel, and P. L. Opresko, "The impact of oxidative DNA damage and stress on telomere homeostasis," Mechanisms of Ageing and Development, vol. 177, pp. 37-45, 2019.

[26] C. Ledda, E. Cannizzaro, P. Lovreglio et al., "Exposure to toxic heavy metals can influence homocysteine metabolism?," Antioxidants, vol. 9, no. 1, p. 30, 2020.

[27] D. Jurk, C. Wilson, J. F. Passos et al., "Chronic inflammation induces telomere dysfunction and accelerates ageing in mice," Nature Communications, vol. 5, no. 1, p. 4172, 2014.

[28] J. Liu, M. Xu, L. Ding et al., "Prevalence of hypertension and noise-induced hearing loss in Chinese coal miners," Journal of Thoracic Disease, vol. 8, no. 3, pp. 422-429, 2016.

[29] X. X. Liu, Y. Lu, H. Z. Liu, and Y. He, “Correlation between telomere length of peripheral blood leukocytes and noiseinduced hearing loss," China Academic Journal, vol. 3, pp. 223-225, 2017.

[30] M. B. Hosseinabadi, N. Khanjani, T. Münzel, A. Daiber, and M. Yaghmorloo, "Chronic occupational noise exposure: effects on DNA damage, blood pressure, and serum biochemistry," Mutation Research/Genetic Toxicology and Environmental Mutagenesis, vol. 841, pp. 17-22, 2019.

[31] K. Lu, R. P. Abo, K. A. Schlieper et al., "Arsenic exposure perturbs the gut microbiome and its metabolic profile in mice: an integrated metagenomics and metabolomics analysis," Environmental Health Perspectives, vol. 122, no. 3, pp. 284-291, 2014.

[32] A. Benetos, K. Okuda, M. Lajemi et al., "Telomere length as an indicator of biological Aging," Hypertension, vol. 37, no. 2, pp. 381-385, 2001.

[33] T. B. Opstad, A. A. Kalstad, A. Å. Pettersen, H. Arnesen, and I. Seljeflot, "Novel biomolecules of ageing, sex differences and potential underlying mechanisms of telomere shortening in coronary artery disease," Experimental Gerontology, vol. 119, pp. 53-60, 2019.

[34] B. L. Needham, A. V. Diez Roux, C. E. Bird et al., "A test of biological and behavioral explanations for gender differences in telomere length: the multi-ethnic study of atherosclerosis," Biodemography and Social Biology, vol. 60, no. 2, pp. 156$173,2014$.

[35] P. T. Williams, "Increases in weight and body size increase the odds for hypertension during 7 years of follow-up," Obesity, vol. 16, no. 11, pp. 2541-2548, 2008.

[36] J. Huzen, L. S. M. Wong, D. J. Veldhuisen et al., "Telomere length loss due to smoking and metabolic traits," Journal of Internal Medicine, vol. 275, no. 2, pp. 155-163, 2014.

[37] M. Roerecke, J. Kaczorowski, S. W. Tobe, G. Gmel, O. S. M. Hasan, and J. Rehm, "The effect of a reduction in alcohol consumption on blood pressure: a systematic review and meta- analysis," The Lancet Public Health, vol. 2, no. 2, pp. e108e120, 2017.

[38] L. Gang and H. Xiaohong, "GW25-e0837 Effects of tea intake on blood pressure: a meta-analysis of 21 randomized controlled trials," Journal of the American College of Cardiology, vol. 64, no. 16, p. C112, 2014.

[39] Y. Wang, F. Xing, R. Liu et al., "Isolated diastolic hypertension associated risk factors among Chinese in Anhui Province, China," International Journal of Environmental Research and Public Health, vol. 12, no. 4, pp. 4395-4405, 2015.

[40] R. M. Cawthon, "Telomere measurement by quantitative PCR," Nucleic Acids Research, vol. 30, no. 10, pp. 47e-447, 2002. 